\title{
Machine vision systems for on line quality monitoring in industrial applications
}

\author{
Giuseppe Di Leo, Consolatina Liguori, Alfredo Paolillo, Antonio Pietrosanto \\ Department of Industrial Engineering, University of Salerno, via Giovanni Paolo II, 132 Fisciano (SA), Italy
}

ABSTRACT

This paper is concerned with the topic of non-contact measurements for industrial applications. It presents the case of vision-based systems realized by the authors expressly for the measurement of geometric and/or chromatic parameters of rubber profiles for the automotive industry. After a brief description of the extrusion process, a stereo vision system for the on-line measurement of the dimensional characteristics of the profile transversal section is first described in all its main components and features, then two modules for the inspection of the finish of profile surfaces are presented.

\section{Section: RESEARCH PAPER}

Keywords: machine vision, non-contact measurement, image processing, gloss, color image, stereo vision

Citation: G. Dileo, C. Liguori, A. Paolillo, A. Pietrosanto, Machine vision systems for on line quality monitoring in industrial applications, Acta IMEKO, vol. 4, no. 1 , article 18, February 2015, identifier: IMEKO-ACTA-04 (2015)-01-18

Editor: Paolo Carbone, University of Perugia

Received January $15^{\text {th }}, 2014$; In final form November $17^{\text {th }}, 2014$; Published February 2015

Copyright: (c) 2014 IMEKO. This is an open-access article distributed under the terms of the Creative Commons Attribution 3.0 License, which permits unrestricted use, distribution, and reproduction in any medium, provided the original author and source are credited

Funding: (none reported)

Corresponding author: Giuseppe Di Leo, e-mail: gdileo@unisa.it

\section{INTRODUCTION}

Thanks to new technologies that today allow to implement automatic nonintrusive solutions at convenient costs, on line inspection systems are going to be widespread also in areas driven by off-line quality control requirements. Dimensions and external finish of products can be today monitored on-line at costs that can be recovered after few months of production waste reduction. A number of inspection techniques have been thought to be implemented on-line and the choice among them must be made on the basis of the product nature. Non-contact measurements are strongly recommended in many applications, e.g. to avoid load effects when deformable objects are to be inspected. Machine vision methods have provided the efficient and reliable techniques for the design of image-based non-contact measurement systems [1].

In some industrial fields, such as the production of either electronic components and boards or mechanical parts, automated inspection using machine vision has already had a relevant number of applications. This is not the case of the production of rubber profiles, due to the complexity of the production process and to the flexibility and the dark color of the product.

In this field the authors realized an image-based measurement system for inspection tasks in a plant producing rubber profiles for the automotive industry [2][4]. A stereo vision system was designed to make the on-line contour extraction, the $3 \mathrm{D}$ reconstruction of the transversal section of rubber profiles, and the measurement of the main dimensional parameters. It is based on the registration of each observed profile onto a reference profile. A suitable software tool let users specify the dimensional lengths to be measured. In this way, users have been able to maintain the software up to date for new models of profiles and new measurements [2]. Nevertheless the measurement system still keeps some limits: i) the robustness of the contour extraction algorithm gets low when a metal reinforcement is included in the profile section; ii) the software does not provide statistical information on the product quality; iii) no inspection is made on the external surface of the profile. In this paper which is an extended version of [3] the authors, after a brief recall of the overall structure of the extrusion line, describe the solutions they propose to 


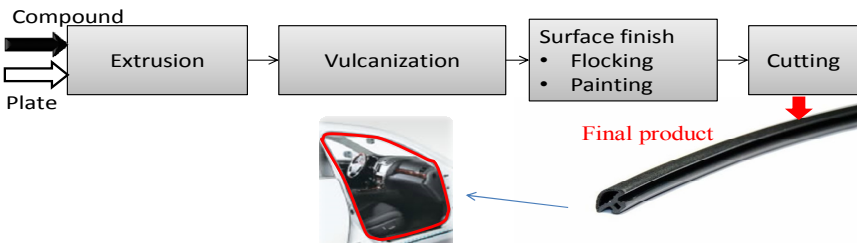

Figure 1. Stages for the rubber manufacturing.

overcome the aforementioned limits of the contactless measurement system.

\section{THE EXTRUSION LINE}

At the site of Battipaglia, Italy, the Cooper Standard Automotive S.p.A. manufactures rubber profiles for car window and door sealings. The production cycle is divided into several basic steps (see Figure 1). The extrusion essentially compresses the pasty material at high pressures through a matrix which reproduces the interior and exterior shape of the piece. Some profiles, the so-called armed profiles, have a metal insert with stiffening function. The extruded rubber flows through ovens for the vulcanization which causes a change in the molecular structure of the polymer resulting in an increase of the elasticity and tensile strength and in an improvement of other physical and chemical properties. Subsequently, the product passes to the phase of electrostatic flocking which gives the rubber a velvety appearance, obtained by first sprinkling an adhesive solution over the surface and then uniformly distributing fibers over it by means of an electrostatic field. After a cooling step, those products for which it is required undergo a brushing step. This phase has a predominantly aesthetic purpose so as to give it a matt appearance to part of the outer surface. The step of cutting to a given length ends the in-line processing, followed by the collection of the products in the cases for the transport.

\section{THE MEASUREMENT SYSTEM}

The measurement station for the verification of the geometrical dimensions of the profile has been placed at a side of the second conveyor belt used for the collection of the finished product (Figure 2): It is constituted by two cameras, an illuminator, a photocell and a data acquisition system. The cameras are suitably positioned to frame the cross section of the profile with different angles of view. The on-line operation of the measurement station can be viewed as a sequence of modules, detailed in the following and shown in Figure 3. The main module is the on-line procedure, which is executed each time a new profile goes into the visual field of the two cameras. Both (left and right) images feed the $2 \mathrm{D}$ procedure which outputs the edge points of the two contours. Then, starting from the contours of the two 2D images, a 3D reconstruction [5]-[6] of the section of the profile is run. In order to determine the position in the space of the profile points of the profiles from the pair of images, taken by the two cameras, it is necessary to know the parameters of the intrinsic and extrinsic cameras, obtained by means of a calibration procedure [7]. The measurements are carried out on the three-dimensional reconstruction by a third module of the developed application, called the Profile Editor.

\subsection{Two-dimensional image processing}

Since the measurements are made on the 3-D reconstruction of the $2-\mathrm{D}$ contours, the localization of the cross-section contour plays a key role in the measurement procedure. The developed image processing algorithms apply a sequence of known image processing techniques: a histogram enhancement routine normalizes the grey level distribution in the image, a region growing stage locates all the pixels belonging to the profile section (the foreground region), and a contour tracking algorithm locates the contour as the border of the foreground region. For the profiles with metal reinforcement, the tuning of parameters such as integration time, lens aperture and amplifier gain of the camera becomes critical as they have a direct influence on the brightness of the captured image. If a value of these parameters is found so that the rubber part of the front section is represented with a sufficiently high grey level, the metal sheet, which has a much higher reflectance, saturates (i.e. reaches the maximum grey level) and generates glares, smearing or blooming broadening the appearance of the lit

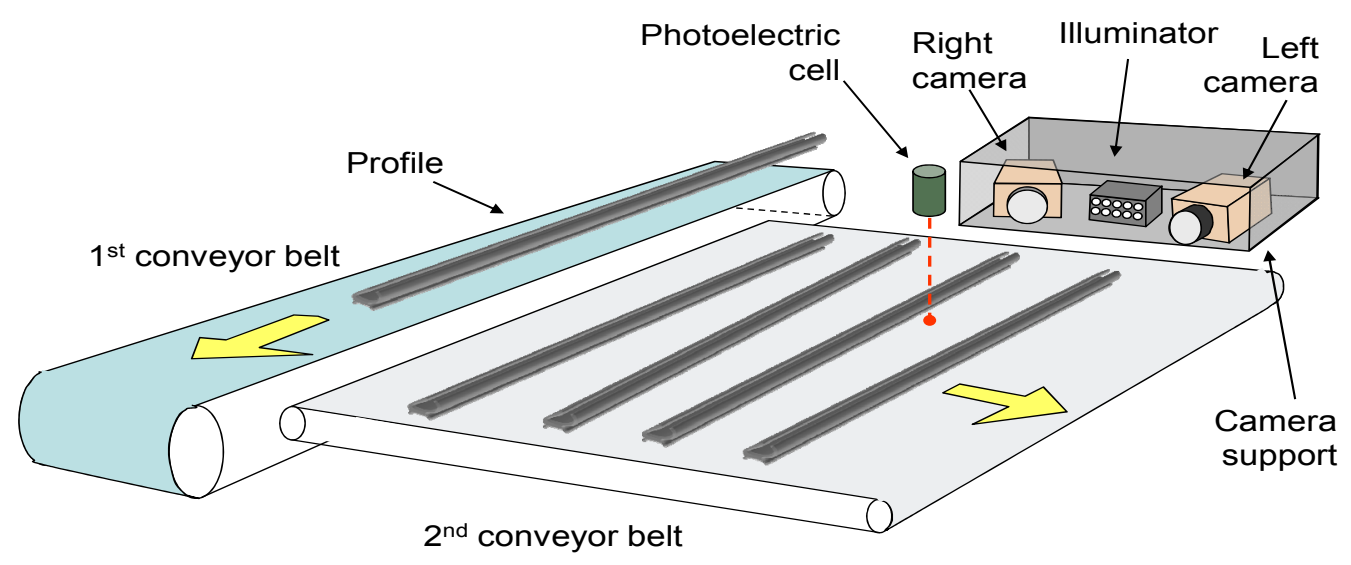

Figure 2. The hardware architecture of the measurement station. 


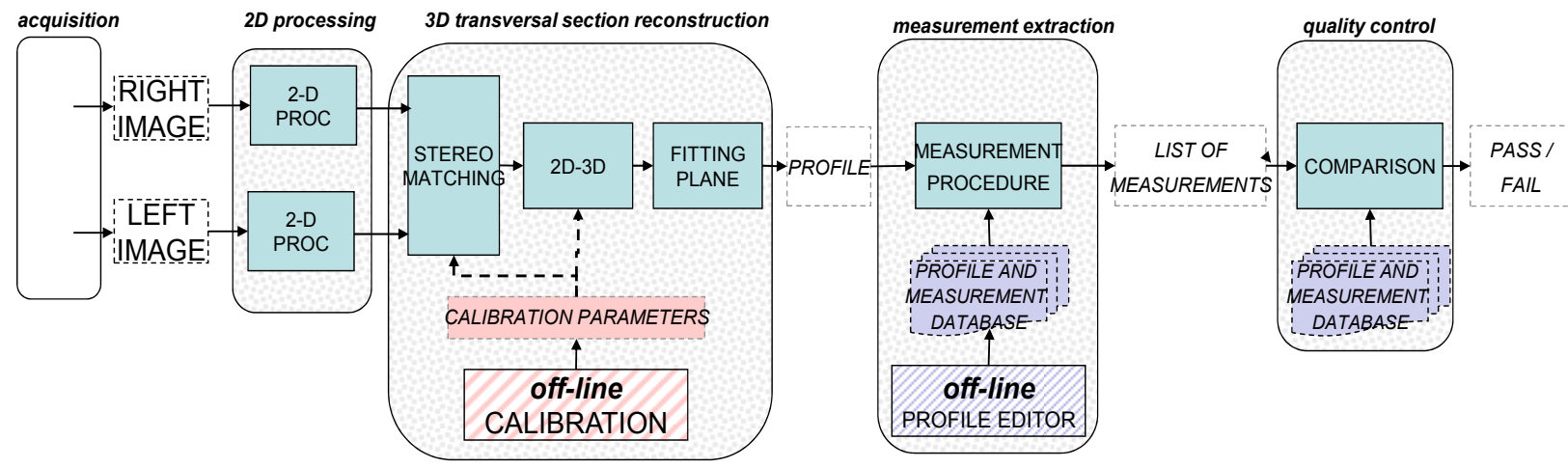

Figure 3. The software architecture.

part and affecting the measurement.

In order to obtain high-contrast and to avoid these undesirable phenomena, the dynamic range of the image on which measurements have to be done must be expanded. Two techniques were considered: the High Dynamic Range (HDR) imaging [8] and Exposure Fusion (EF) [9], which, starting from a sequence of images of the same scene taken with different exposures, reconstruct an image with a higher dynamic range. The HDR technique aims to reconstruct the radiance map of the scene: from the sequence of images at different exposures, the characteristic function (CRF, camera response function) which relates the radiance of the scene to the brightness of individual pixels is first reconstructed. Then by inverting this function the radiance map of the scene can be achieved. The HDR

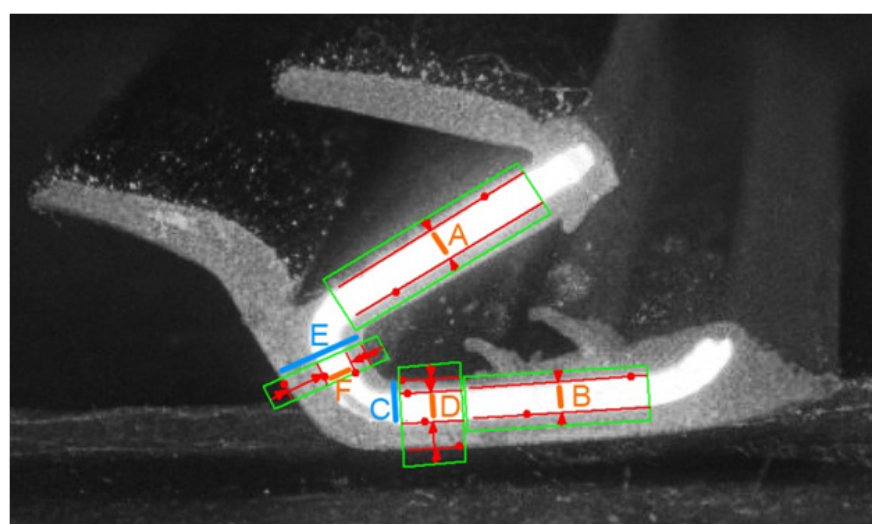

a)

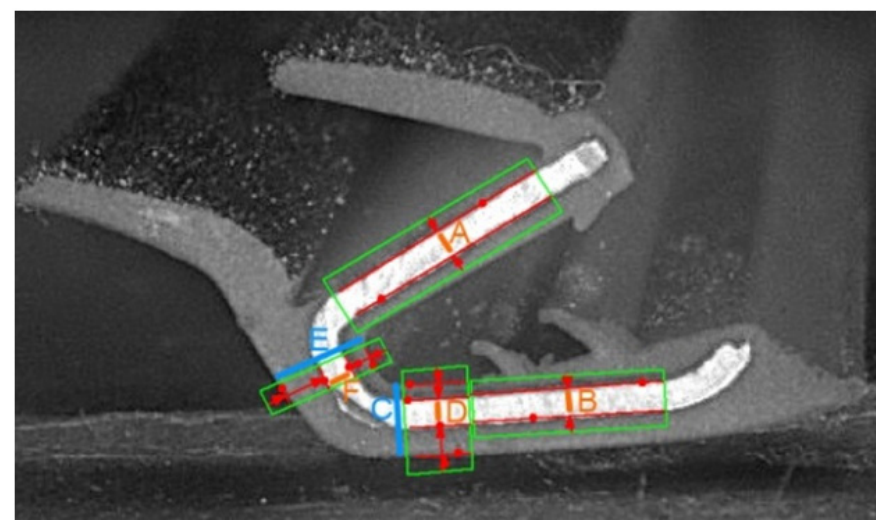

b)

Figure 4. a) HDR image with overlaying measurement indicators; b) enfused image with overlaying measurement indicators. processing, however, imposes a significant computational load that is not compatible with the requirements of on-line control. Furthermore, the images obtained with this technique tend to create undesirable halos around bright areas of the image, and then the presence of reflections on the metal part would cause a broadening of the contours. The EF technique addresses some of the limitations of HDR: The final image is composed by taking the individual pixels from images of the sequence on the basis of a score, according to specific figures of merit associated to the individual pixels of each image. By this way, two goals can be achieved: i) the execution of the algorithm is much faster, because there is no need to sort the map of radiance again; ii) the effect of halos that was observed in HDR processed images is eliminated, thus allowing a more accurate contour extraction. Figures 4a) and b) show the images obtained with the two techniques mentioned above and the related measures. In both cases the rubber part is measured correctly, while the measurements of the metal part have a maximum error of $21 \%$ compared to the reference for the image obtained by means of HDR, and 6\% in the case of images obtained via EF, thus demonstrating the effectiveness of the EF technique in preserving the outlines of the brighter areas.

\subsection{Three-dimensional reconstruction}

The two 2-D contours determined in the previous step are processed in order to obtain a 3-D reconstruction of the contours of the leading cross-section. The procedure can be divided into two phases: i) the search for matching stereo pairs, namely the pair of image points in the left and right images generated by the same real point, and ii) the calculation of the 3-D coordinates of the profile contour points as a function of the two stereo pairs. The search for stereo pairs is performed by exploiting the epipolar constraint [6]: given a point on one image (e.g. the left one), the corresponding point on the other (e.g. the right) image lies on a line, the so-called epipolar line, whose localization can be determined from the calibration parameters. The point on the right image which corresponds to a given point on the left image can be searched for within the intersections between its epipolar line and the profile contours. Once the calibration parameters of the two cameras and the pixel coordinates of two image points are 


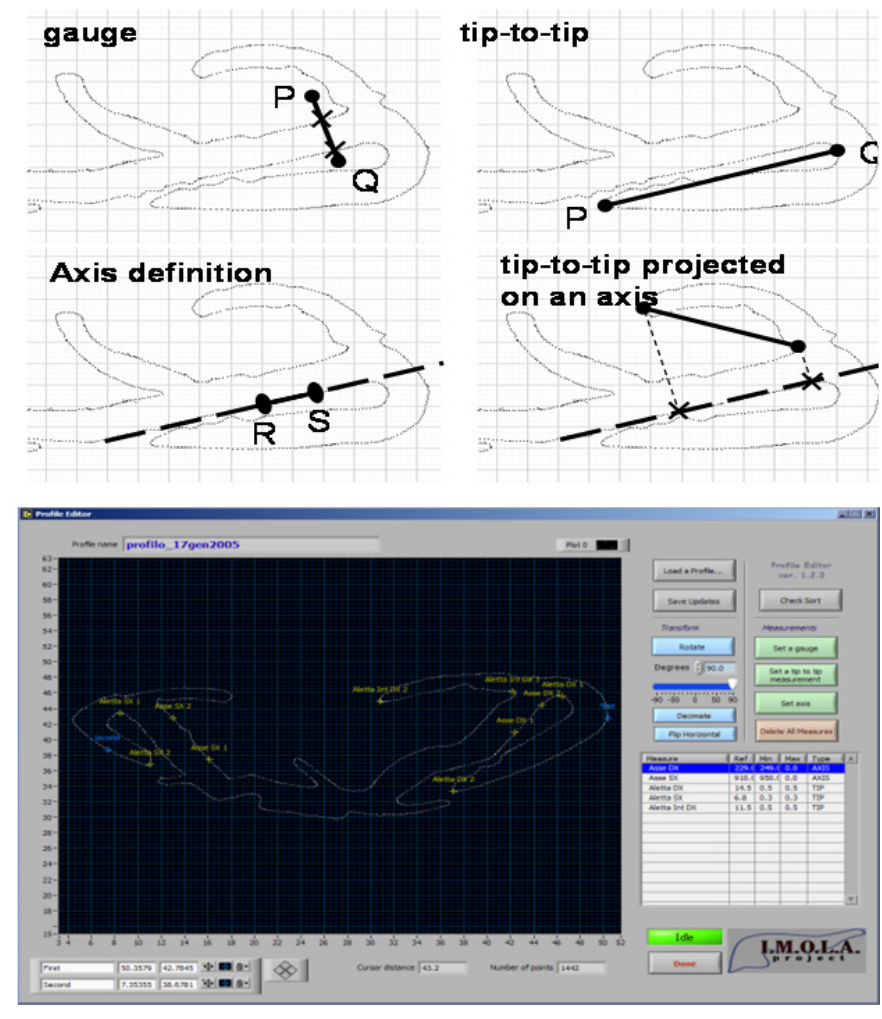

Figure 5. a) Measurement primitives; b) example of use of the Profile Editor.

known, a triangulation algorithm [5] allows to obtain the measurement of the three absolute coordinates of each point of the contour. In order to simplify the subsequent extraction of measurements from the $3 \mathrm{D}$ contour, a fitting plane is determined as the least square plane approximating the points of the cross section of the profile, and then the measurements are performed on the 2-D projection of the contour on this plane.

\subsection{Measurements}

A procedure of measurement valid for all the profiles and products has been implemented and set up. The procedure can also be extended to future production, since the list and the description of the measurements can be easily tailored for a new model of profile. The dimensions to be measured must be only once specified by the user on the coordinate system of the reference profile contour. Then, the measurement procedure consists of two main steps: i) each new observed $3 \mathrm{D}$ contour of the profile is superimposed ("registered") to the reference contour of the profile; ii); the measurements specified on the reference are made on the observed contour.

The reference profile library management is entrusted to another application module, called Profile Editor. This module allows the user to select and view the reference profile of a section, to specify the measurements to be made on the profile. The measurements are defined by primitives, each of which requires the user to define two points on the reference contour; these couples of points must be placed in the coordinate system of the reference contour. The different types of measurement primitives that can be applied to profiles are:
- the "gauge", distance between the two intersections of a specified segment $(\mathrm{P}, \mathrm{Q})$ and the observed profile;

- the so-called "tip-to-tip", distance between the two points of the profile observed that best correspond, in terms of proximity and curvature, to two specified points on the reference profile (P, Q). It is also possible to project the measured segment onto a straight line ("axis") determined by two points belonging to the contour ( $R$ and $\mathrm{S}$ of Figure $5 \mathrm{a}$ ) and specified using the Profile Editor. For each selected measurement the nominal value can be stored in the data base and compared on line with the measured one. In Figure 5b) a screen output of the software is shown.

\subsection{Calibration}

In order to determine the spatial position of the profile contour points the three-dimensional reconstruction algorithm requires that the intrinsic and extrinsic parameters of the cameras are known. Goal of the calibration procedure is the determination of the intrinsic and extrinsic parameters of the cameras. It must be repeated whenever one of the configuration parameters of the system (position or angle of the cameras, distance, focus) are expected to have changed. The authors' proposal is based on the approach of Zhang [7], which requires the two cameras to observe a different planar target, whose geometry in 3-D space is known with very good precision, shown at a few different unknown poses. A sandblasted metal plate containing a pattern of $6 \times 5$ square holes $(120$ squares corners) is adopted as the target, due to its good accuracy $(\sim 10 \mu \mathrm{m})$. The knowledge of the corner positions in several pairs of images, at least five, and of the target geometry in $\mathrm{mm}$, allows specific calculations based on the maximum likelihood criterion in order to determine the intrinsic and extrinsic parameters of the cameras.

\subsection{Quality checks, report and metrological characterization}

Each measurement result is compared with the specification limits in order to assess its compliance with design tolerances and the software reports the outcome of the dimensional test with a "pass/fail" indication. The presentation of the results includes, for each piece, the superposition of the observed and the reference profiles, the table of last results, the time chart of the results and some statistics. Some report pages can be recalled to view the chart of the quantities of interest. The user's queries can include: the line, the section, date and time of beginning and ending of observation, and the quantity of interest. Summary reports of different types can be generated: i) Basic, ii) Advanced, iii) Failure Chart, iv) Production Line, v) Summary. The metrological characterization was carried out on the system in use at Cooper Standard Automotive plant in Battipaglia, Italy. Tests have been performed to assess: (i) the influence of the relative position of the cameras, (ii) the systematic errors of the system, and (iii) the repeatability of the measurement results. Choosing the optimal position of the cameras and after performing a correction of systematic errors, the standard uncertainty [10] of all types of measurements was contained in $0.2 \mathrm{~mm}$. The processing time of the proposed measurement system, 
(a)

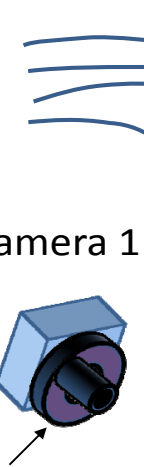

Illuminator

(b)

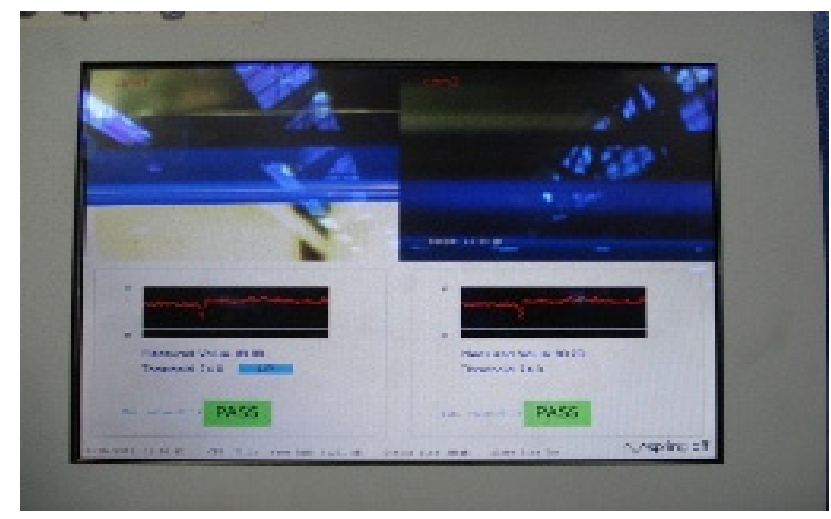

Figure 6. a) Painting quality measurement system; b) software user interface.

at the typical extrusion speed of the line ( 3 pieces / min), allows to monitor the full $100 \%$ of the produced pieces.

\section{A SYSTEM FOR PAINTING QUALITY CONTROL}

The coating process consists in depositing a transparent silicone paint on the exterior surface of the profile. The coating has both an aesthetic and a functional purpose: to confer a shiny effect and to make a waterproof seal.

The coating system in use at the plant is produced by Kremlin, consists of four air spray guns, and is managed by a PLC, which allows to set for each gun both the pressure of the paint and of the air. The system for the regulation of the pressure is based on the use of electronic controllers, which allow to obtain a great precision in regulating the value of air flow and paint. This results in an excellent finish quality with a very high degree of pulverization, and in a very low thickness of the painting (from $10 \mu \mathrm{m}$ to $30 \mathrm{~m})$. Nevertheless, temporary or permanent gun failures may result in parts not painted that must be detected and discarded. Since the paint is transparent, in order to allow visual verification by the operator, it is mixed with an additive (UVITEX OB by CIBA) which emits in the visible when illuminated with ultraviolet (UV) radiation. In the previous system, a UV lamp illuminated the profile, a CCTV system grabbed images of that profile and showed them on a monitor, where a human operator had to periodically check whether the profile image had the required brightness. The vision system designed by the authors replaces the human operator in the control of paint. It has been designed to estimate the amount of paint deposited on the surface of the profile in order to detect possible faults on the air spray guns. The vision system, schematically shown in Fig 6a), is positioned immediately after the spray booth and uses two camera-illuminator pairs in order to monitor the whole external surface that must be painted. The two $640 \times 480$ color cameras are featured with ring type illuminators having a wavelength of $365 \mathrm{~nm}$. The system acquires at regular time intervals two images of the profile surface and then processes them to measure the amount of paint and compare it with a predefined threshold. The image processing is based on the measurement of the amount of blue color present in each acquired image, since it is proportional to the amount of additive and then of paint. In particular, the algorithm identifies a sub-image centred onto the profile contour, and calculates an index $M$ proportional to the average brightness of the blue component extracted from the acquired sub-image. The profile contour is located by searching the first and last edge along vertical lines with a differential operator. The control panel (Fig. 6b) contains the processing unit, the monitor for display, the stack lights, acoustic signalling devices and the power supply plugs. If the measured value is out of tolerance, an alarm is generated. A chart is also shown on the operator panel with the history of the last 300 measurements. In this way, the operator can periodically monitor the measurements and anticipate unwanted trends.

\subsection{Assessment of fault detection capability}

Tests have been carried out in order to verify the functionality of the system and the compliance to production needs. Faults on each gun and multiple faults are simulated obstructing the guns and the indices measured on the images of the two cameras are recorded. For each kind of fault, the fault state is maintained for about 30 seconds and the variability range of the indices is recorded. This whole procedure is repeated 3 times. Said P1, P2, P3 and $\mathrm{P} 4$ the status of the 4 guns and M1 and M2 the two indices measured on the images from the two cameras, Table 1 reports the minimum and maximum observed value during the tests for the main fault cases (single or multiple) and for unfaulty conditions. As can be seen, the sensitivity to the spray gun fault is different for the two parameters (M1, M2), due to the positioning of the four air spray guns and the two cameras, as shown in Fig. 6a. Moreover, different absolute values happen to be measured in unfaulty condition, since different types of profiles have different shapes and then respond to the illumination differently. Analyzing the results, the unfaulty condition appears clearly separate from the single faults, that are the most difficult to detect. The diagnostic capability is acceptable since it is possible to identify, at least, on which side of the profile there is one or more guns not working correctly. 


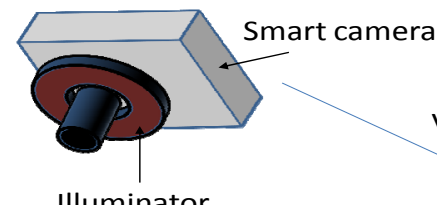

Illuminator

(a)

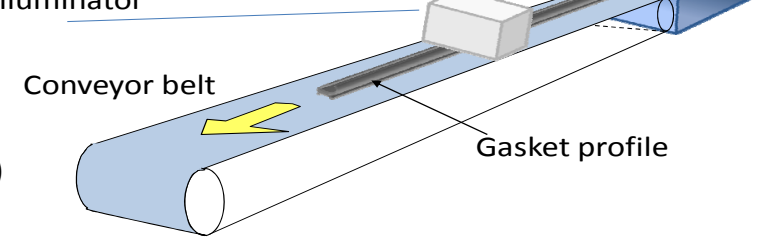

(b)

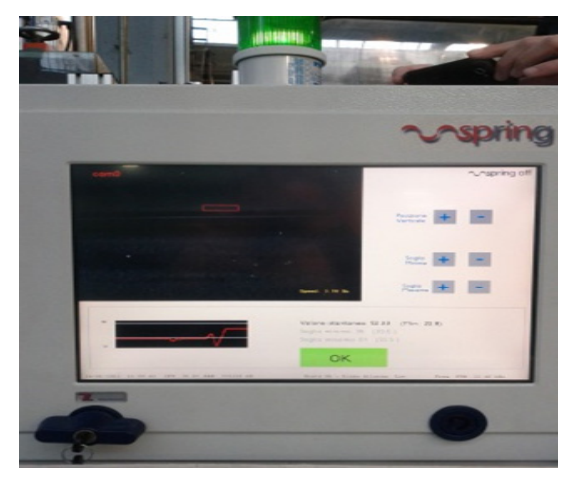

Figure 7. a) Gloss measurement system; b) software user interface.

Other tests are carried out in order to verify the system sensitivity to partial obstruction of a paint spray gun. In this case the system detects the fault only when the obstruction is relevant.

Finally, tests with a complete obstruction of a single gun for a short time period are performed. The promptness of the detection is related to the measurement rate; the time required to process a couple of images is about $100 \mathrm{~ms}$. Since the maximum line speed is $20 \mathrm{~m} / \mathrm{min}$, and the field of view is about $10 \mathrm{~cm}$, each measurement covers a length of about $3 \mathrm{~cm}$. Then, the system is able to detect the lack of paint for length at least greater than $6 \mathrm{~cm}$. These characteristics fully respond to specific requests of the factory quality control system.

\section{ON-LINE GLOSS METER}

One of the final stages of the production line is the brushing, where a rotating brush can be moved vertically in order to abrade with a different intensity the surface of the profile and then to give a different matt appearance to the surface. Also this stage has a predominantly esthetical purpose. In order to verify the obtained matt appearance, the degree of gloss (expressed in gloss unit, GU) was traditionally measured off-line on some samples using a commercial gloss-meter. These measurements are then compared with the gloss acceptance range [1.5 - 3.5 GU]. The gloss measurement exploits the phenomenon of specular reflection of a surface illuminated by a light beam. The gloss-meter projects a non-polarized white light on the sample surface, at a predetermined angle of incidence and it measures the intensity of the reflected light with a sensor
Table 1 . Test results for faulty and unfaulty cases.

\begin{tabular}{cccc|c|c}
\hline P1 & P2 & P3 & P4 & M1 & M2 \\
\hline Ok & Ok & Ok & Ok & $80-90$ & $65-80$ \\
Fault & Ok & Ok & Ok & $40-65$ & $50-75$ \\
Ok & Fault & Ok & Ok & $40-60$ & $50-80$ \\
Ok & Ok & Fault & Ok & $80-90$ & $20-30$ \\
Ok & Ok & Ok & Fault & $75-90$ & $25-40$ \\
Fault & Fault & Ok & Ok & $30-45$ & $50-80$ \\
Ok & Ok & Fault & Fault & $70-85$ & $15-25$ \\
Fault & Fault & Fault & Fault & $10-20$ & $10-20$ \\
\hline
\end{tabular}

located in a specular position with respect to the light source. According to the standard [11], the angles of incidence for the gloss measurement are either $20^{\circ}, 60^{\circ}$ or $85^{\circ}$ depending on the gloss value. The off-line measurements show several drawbacks: they require a relevant time, they cannot be carried out on all the pieces, and specialized operators are required.

The research activity has been aimed at achieving an online non-contact gloss-meter. The approach suggested by the standard [11] cannot be precisely followed. The incidence and reflection angles of light rays cannot be accurately controlled online, since the rubber profile can have small movements and its surface is generally not flat. The proposed technique uses a white LED illuminator ring (Advanced Illuminator RL127) as the light source, and a digital smart camera (NI 1772, $640 \times 480$ pixels, 8 bit, 110 fps) as the sensing device [12].

The measurement station is positioned immediately after the brushing booth (Fig. 7a). The smart camera is positioned on top of the profile and acquires an image of a portion of the profile. The processing steps applied onto the acquired image are the following: i) the position of the profile within the image is determined by edge detection; ii) a sub-image of $30 \times 20$ pixels is located at the centre of the detected profile; iii) the average brightness of the sub-image is evaluated. The measured value can be related to the gloss value through a linear relationship that is experimentally estimated with a calibration procedure described in section 5.1. The measured gloss is compared with a tolerance value defined by the product specifications. If the measured value is out of tolerance, an alarm is generated.

The operator panel (Fig. 7b) shows the acquired image, the overlay of the sub-image rectangle, the measured gloss value and a chart with the history of the last 300 measurements.

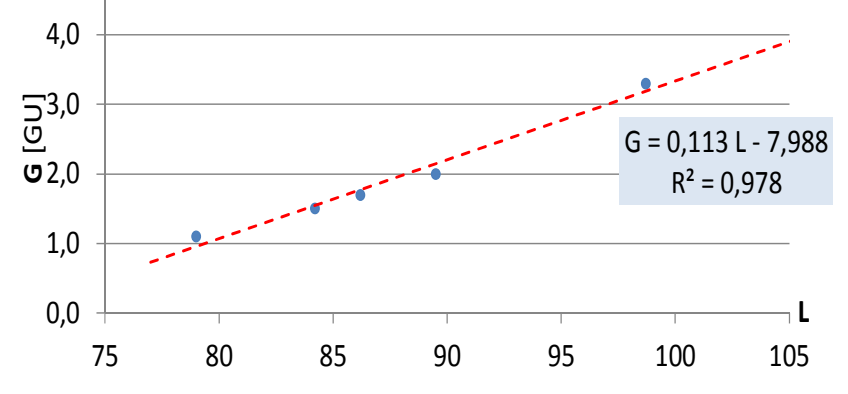

Figure 8. The results of calibration. 


\subsection{Calibration procedure}

A calibration procedure is required in order to estimate the relationship between the average brightness of the subimage and the gloss value. As a reference instrument, an Erichsen Pico-Glossmaster 500 is used, since it is the instrument currently in use at the quality laboratory of the plant for offline measurements. It has a measurement range of $[0,100] \mathrm{GU}$ and an accuracy equal to $0.2 \mathrm{GU}$.

The following steps of the calibration procedure are repeated for $N$ different brush positions:

- The brush is set in a fixed position.

- The average brightness of the sub-image is measured for 30 seconds. Mean $(L)$ and standard deviation $(L)$ of the averages are evaluated. While the profile is being measured the starting and final point are marked on its surface.

- The marked profile length is measured offline with the reference instrument, by taking 30 uniformly spaced measurements. Mean $(G)$ and standard deviation $(\sigma G)$ of these measurements are evaluated.

A linear fit is applied on the pairs $\left(G_{i}, L_{i}\right)$ in order to estimate the calibration curve. Figure 8 shows the calibration points, the obtained curve, its analytical formulation and the determination factor. Being $\mathrm{R}^{2}$ equal to 0.978, the linear approximation can be considered acceptable. The standard deviation $Y$ of the output of the linear fit model can be estimated with the following relationship:

$$
\sigma_{Y}=\sqrt{\frac{1}{N-1} \sum_{i=0}^{N-1}\left(G_{i}-\widehat{G}_{i}\right)^{2}}
$$

where $\hat{G}_{i}$ is the value obtained with the linear approximation at the abscissa $L_{i}$.For almost all the calibration points the standard deviation $L$ is practically the same in all points and nearly equal to 4 . The standard deviation $Y$ is equal to $0.2 \mathrm{GU}$. The uncertainty [10] of final gloss measurements can be estimated with the following relationship:

$$
u_{G}^{2}=\left(s \cdot \sigma_{L}\right)^{2}+\sigma_{Y}^{2}
$$

where $s=0.113$ is the slope of fitting line, and resulted to be equal to $u_{G}=0.5 \mathrm{GU}$.

As far as the measurement rate is concerned, since the system is able to process about 4 images per second and the maximum speed of the line is equal to $20 \mathrm{~m} / \mathrm{min}$, the system is in able to perform a measurement every $8 \mathrm{~cm}$. The obtained metrological characteristics meet the requirements of the quality control system.

\section{CONCLUSIONS}

A vision based system for the on-line measurement and control of dimensional and esthetical parameters of rubber profiles has been designed and characterized by the authors. The measurement system has demonstrated to be efficient and to fully meet the specifications of the industrial quality production control system. Moreover, this on-line noncontact solution has caused a drastic reduction of production waste, and then a decrease of costs.

The systems presented in this paper are going to be connected to the same fieldbus plant network. In this way a supervising application, which is under development, will allow to monitor the status of the entire production line, to save results into a database, to manage report printing, to coordinate the feedback actions.

\section{REFERENCES}

[1] Te-Hsiu Sun, Chun-Chieh Tseng, Min-Sheng Chen, "Electric contacts inspection using machine vision" Image and Vision Computing, Volume 28, Issue 6, June 2010, Pages 890-901.

[2] R. Anchini, G. Di Leo, C. Liguori, A. Paolillo, "Metrological Characterization of a Vision-Based Measurement System for the Online Inspection of Automotive Rubber Profile”, IEEE Transactions on I\&M, Vol. 58, No. 1, Jan. 2009, pp. 4-13.

[3] G. Di Leo, C. Liguori, A. Paolillo, A. Pietrosanto, "Contactless measurements for on line quality monitoring in rubber extrusion processes”, 19th Symposium IMEKO TC 4 Symposium and $17^{\text {th }}$ IWADC Workshop Advances in Instrumentation and Sensors Interoperability July 18-19, 2013, Barcelona, Spain pp. 197-202.

[4] G. Di Leo, C. Liguori, A. Paolillo, "Covariance propagation for the uncertainty estimation in stereo vision", IEEE Transactions on Instrumentation and Measurement, Vol. 60, Issue 5, DOI 10.1109/TIM.2011.2113070, accepted for publication.

[5] R. I. Hartley and P. Sturm, Triangulation, Comput. Vision Image Understand., 68-2 (1997-11), 146-157.

[6] Hartley, Zisserman, "Multiple View Geometry in Computer Vision", 2nd Ed, Cambridge University Press.

[7] Z. Zhang, "A flexible new technique for camera calibration", IEEE Transactions on Pattern Analysis and Machine Intelligence, 22(11):1330-1334, 2000.

[8] E. Reinhard, G. Ward, P. Debevec, W. Heidrich et al., "High Dynamic Range Imaging: acquisition, display and imagebased lighting”, $2^{\text {nd }}$ ed., 2010, Morgan Kaufmann.

[9] T. Mertens, J. Kautz and F. Van Reeth, "Exposure Fusion”, Pacific Graphics 2007.

[10] JCGM 100:2008, "Evaluation of measurement data - Guide to the expression of uncertainty in measurement".

[11] ISO 2813:1994, "Paints and varnishes - Determination of specular gloss of non-metallic paint films at 20 degrees, 60 degrees and 85 degrees".

[12] T. Aida, S. Hayasaki, T. Sakai, "High Speeding of the GlossMeter for Curved Surfaces Using CCD Line Sensor", Conference on Precision Electromagnetic Measurements, 7-10 June 1988, pp. 359-360. 